\title{
Influence of Localized Surface Plasmon Polaritons on Silver Nanoparticles
}

\section{Cliff Orori Mosiori $^{1 *}$, Walter Kamande Njoroge ${ }^{2}$, Lawrence Otieno Ochoo ${ }^{3}$}

${ }^{1}$ Department of Mathematics and Physics, Technical University of Mombasa, Mombasa - 80100, KENYA

${ }^{2,3}$ Department of Physics, Kenyatta University, P. O. Box 43844 - 00100, Nairobi, KENYA

*Corresponding Contact:

Email: corori@tum.ac.ke

Manuscript Received: 15 April 2020 - Revised: 27 May $2020 \quad$ - Accepted: 10 June 2020

\begin{abstract}
In this article, we present a theoretical study on localized surface Plasmon of spherical Ag nanoparticles (NPs) done by numerical simulation. A plane EM wave was used to determine absorption cross-section and results showed that excitation of LSPPs produced an electric field on the surface of the nanoparticle. This field causes a large cross sectional area that influences higher scattering of incident photon at the surface of an absorber layer. It was concluded that LSPPs excitations in small size spherical particles can be utilized in low-cost solar cells to increase PCE of solar panels and can be expanded to many other fields of optoelectronic technologies ranging from solar cells, through photo diodes to optical bio-sensing applications.
\end{abstract}

Keywords: Polaritons Bio-sensing, Absorption, Extinction, Cross-section, Plasmons, Scattering

This article is is licensed under a Creative Commons Attribution-NonCommercial 4.0 International License.

Attribution-NonCommercial (CC BY-NC) license lets others remix, tweak, and build upon work non-commercially, and

although the new works must also acknowledge \& be non-commercial.

\section{INTRODUCTION}

A surface Plasmon was first explained in terms of spectral reflection using the Mie theory (Petryayeva and Krull, 2011) of light scattering by a metal nanoparticles encountering energy loss (Ritchie, 1957) by travelling electrons in metals. The term "plasmon" describe a free oscillating electron in nanoparticles responsible for energy loss (Mansoor et al., 2018). Energy loss in thin metal nanolayer is due surface Plasmons. Surface Plasmons have a number of applications such as in biosensors, solar cells (Zayats et al., 2003), IR diodes IR spectroscopy (Willets and Van Duyne, 2007) and subwavelength imaging technologies (Hu et al., 2006). Their Plasmon waves propagate either along the surface of a metal layer or inside a layer either at the interface between metal and dielectric or in small size particles comparable to photon wavelengths (Barnes et al., 2003; Billaud, 2007). Plasmons get confined at the metaldielectric interface. In this article, LSPPs of spherical silver nanoparticles are modeled 
numerically to evaluate its effects on a far-field and near-field optical responses and its potential applications as they have exhibited promising influences on nanomaterial semiconductors. In research, nanomaterials are considered to have a number of structures characterized by at least one dimension within 1-100 nm nanoscale range at Petryayeva and Krull, (2011). These nanostructures are used to differentiate between molecules and the bulk systems and determine the distance crystals or particles. Three dimensions are commonly considered as 3D, 2D and 1D to determine the state density of material also referred to the number of energy states per unit volume in a unit energy interval (González et al., 2015).

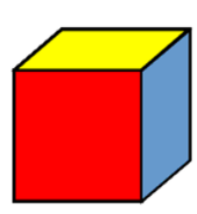

Bulk
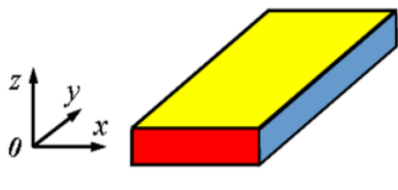

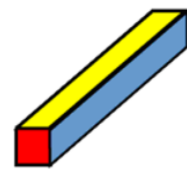

Quantum Wire

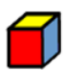

Quantum Dot
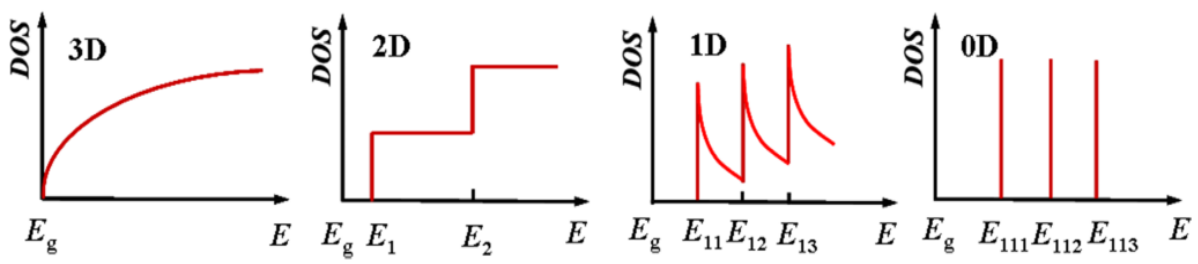

Fig. 1: Representations of 3D, 2D, 1D and OD and density of state-energy curves (Ungureanu et al., 2009)

A semiconductor is classified as nano-material if its system has quantum confinement equal to the Bohr radius within $2-50 \mathrm{~nm}$ range from at least one dimensional approach related as (Nasser, 2017);

$\alpha_{B}=\frac{\hbar \varepsilon}{\mu q^{2}}$

where $\alpha$ represent the radius of nanomaterial. If $\alpha>\alpha_{B}$ then, the system has weak confinement with its density of state proportional to $E^{+\frac{1}{2}}$ while if $\alpha=\alpha_{B}$, then, the system has intermediate confinement with its density of state independent of $\mathrm{E}$. When $\alpha<\alpha_{B}$, the nanomaterial system has strong confinement and the density of state proportion with $E^{-\frac{1}{2}}$ (Nasser, 2017). Confinement in one dimension is called 2D nanomaterial like a quantum wall, in two dimensions is called 1D such as a quantum wire while in three dimensions is called zero dimension 0D nanomaterials like quantum dot. Where $m^{*}$ is effective mass, $E_{c}$ conduction band, $\sigma=1$ or 2 and $\delta$ function peaks. Fig. 1 shows the three types of nanomaterials and their density of states while table 1 shows their energy and density of states for 3D, 2D, 1D and 0D functions. 
Table 1: 3D, 2D, 1D and OD energy and density of states functions

\begin{tabular}{|c|c|c|}
\hline Degrees of freedom & Kinetic energy & Density of states \\
\hline 3D bulk & $E=\frac{\hbar^{2}}{2 m^{*}}\left(k_{x}^{2}+k_{y}^{2}+k_{z}^{2}\right)$ & $\rho_{D O S}^{3 D}=\frac{1}{2 \pi^{2}}\left(\frac{2 m^{*}}{\hbar^{2}}\right)^{3 / 2} \sqrt{E-E_{C}}$ \\
\hline 2D & $E=\frac{\hbar^{2}}{2 m^{*}}\left(k_{x}^{2}+k_{y}^{2}\right)$ & $\rho_{D O S}^{2 D}=\frac{m^{*}}{\pi \hbar^{2}} \sigma \sqrt{E-E_{C}}$ \\
\hline $1 \mathrm{D}$ & $E=\frac{\hbar^{2}}{2 m^{*}}\left(k_{x}^{2}\right)$ & $\rho_{D O S}^{1 D}=\frac{m^{*}}{\pi \hbar} \sqrt{\frac{m^{*}}{2\left(E-E_{C}\right)}}$ \\
\hline $0 \mathrm{D}$ & $E=\frac{\hbar^{2}}{2 m^{*}}$ & $\rho_{D O S}^{0 D}=2 \sigma\left(E-E_{C}\right)$ \\
\hline
\end{tabular}

\section{THEORY}

To analyze LSPP modes, Maxwell's equations are solved. An EM field cause an accumulation of free electrons on one side of a NP, leaving positive charges on the other (Wood, 1902; Catchpole et al., 2008). An electric field is generated opposite to that of the incident photons. This field attempts to restore the electrons to its original equilibrium position resulting into an oscillation with kinetic and electrostatic energies equivalent to that possessed by the incident photon (Kumar et al., 2012; Cao et al., 2014). The excited LSPP inside the particle causes a partial extinction of incident photon as it conserves energy, hence absorption takes place. Scattering cross-section of a spherical metal NP is calculated using (Mansoor et al., 2018);

$$
\sigma_{\text {scatter }}=\frac{8 \pi}{3} k^{4} a_{6}\left(\frac{\varepsilon_{m}-\varepsilon_{d}}{\varepsilon_{m}+2 \varepsilon_{d}}\right)^{2}
$$

where $k$ is the wave vector magnitude of incident light, $\varepsilon_{m}$ is permittivity of the metal and $\varepsilon_{d}$ is permittivity of the surrounding while $a$ is the NP radius. Absorption cross section on the other hand is calculated using Poynting's theorem from:

$$
\sigma_{a b s}=4 \pi k a^{3} \operatorname{Im}\left(\frac{\varepsilon_{m}-\varepsilon_{d}}{\varepsilon_{m}+2 \varepsilon_{d}}\right)
$$

Total extinction cross section is their sum obtained from (Mansoor et al., 2018);

$$
\sigma_{\text {ext }}=\sigma_{a b s}+\sigma_{\text {scatter }}
$$

Therefore, absorption cross-section is a measure of their efficiency and calculating optical absorption is a good approach to determine excitation of the LSPPs of nanoparticles. 


\section{Methodology}

\section{Modeling}

An incident photon field excites free electrons in metal atoms which respond by generating collective oscillations which act as a force that displaces free electrons from positive ions causing resonance behavior (Kumar et al., 2012; Willets and Van Duyne, 2007). To model its action, two metal nanoparticles were illuminated by an incident photon EM field as illustrated in Fig. 1 using model boundaries and was adopted for simulation.

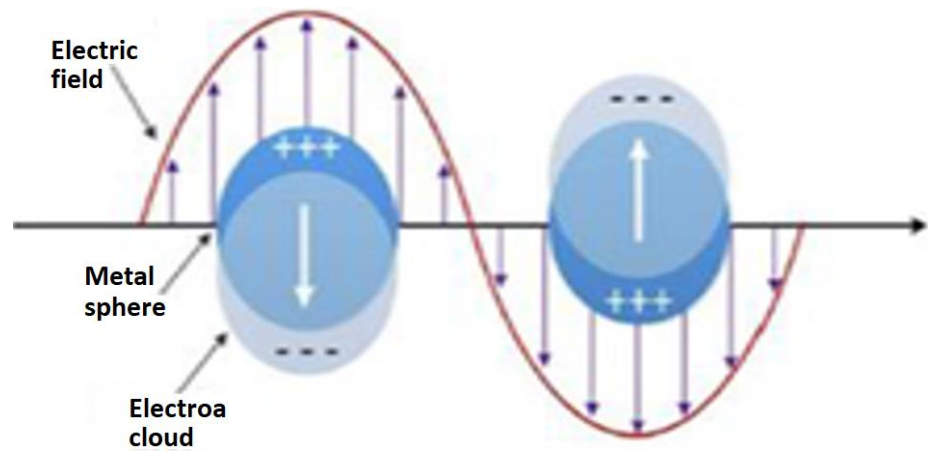

Figure 1: Model of effect of plane wave cause electrons oscillations

\section{CST simulation}

A spherical silver nanoparticle was modeled with a radius of $80 \mathrm{~nm}$ and simulated using frequency domain solver. Electric field distribution on the outer surface and its absorption cross-section were calculated using CST (3D simulation software) exposed to an EM plane wave source as shown in Fig. 2. The CST is a 3D EM solver that solves Maxwell's equation in time domain with Finite Integration Method (FIT) and frequency domain with Finite Element Method (FEM) techniques.

\section{RESULTS}

As shown in Fig. 1, LSPPs are discrete modes as excited by incident photon illuminated in free space. LSPP Frequency modes are dependent on particle geometry and geometrical shape and size determine its resonance frequency modes with a frequency band located in the visible band. Fig. 2 illustrates the electric field distribution as a result of LSPP excitation, while Fig. 3 show simulated absorption cross-section of the $80 \mathrm{~nm}$ silver NP.

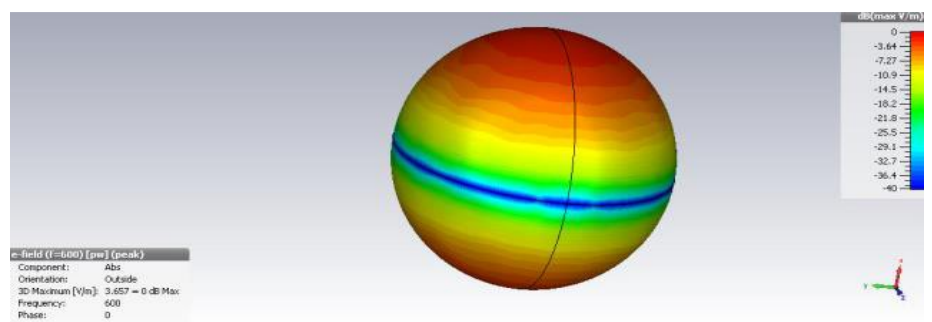

Figure 2. Electric field distribution on the surface of $80 \mathrm{~nm}$ silver spherical NP 


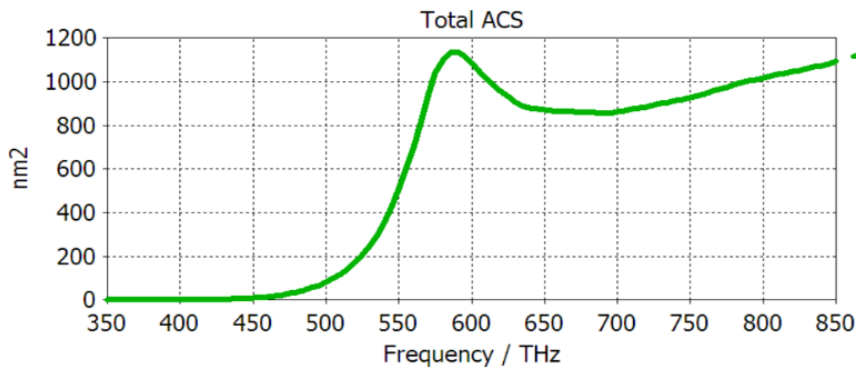

Figure 3. Absorption cross-section of $80 \mathrm{~nm}$ silver spherical NP

As shown in Fig. 2, LSPP excitation results into an increased electric field strength at NP surfaces while Fig. 3 shows an increased absorption as a result of light coupling with surface Plasmons. Similarly, Fig. 2 exhibit a relative agreement with Fig. 1. Therefore, if a NP absorbs half of incident photons that hits its surface, its absorption geometrical factor (Petryayeva and Krull, 2011) reduces by half. The incident photons gets scattered in different directions which can be evaluated. In the silver $(\mathrm{Ag})$ nanoparticles, coherence of oscillations of free electrons coupled incident photon energy to cause the electrons to oscillate in the conduction band (Mansoor et al., 2018; Zayats et al., 2003; Willets and Van Duyne, 2007; Tokman et al., 2000; Catchpole et al., 2008; Billaud, 2007; Cao et al., 2014). This caused the excitation of Surface Plasmon Polaritons (SPPs) (Ungureanu et al., 2009). In fact, localized Surface Plasmon is prominent optical property exhibited by small size metallic nanoscale particles (Barnes et al., 2003; Ritchie, 1957; Kumar et al., 2012; Pattnaik and Priyabrata, 2005) and their extinction cross-section is of great interest in evaluating the performance of LSPPs (Pattnaik and Priyabrata, 2005; Mansoor et al., 2018; Zayats et al., 2003; Willets and Van Duyne, 2007).

\section{CONCLUSION}

In this study, localized surface Plasmon of spherical Ag NPs were numerically simulated and presented. Their absorption cross-section under illumination by a plane EM wave was determined. It was noted that LSPPs excitation caused an electric field to be established on the surface of a nanoparticle resulting into a large scattering cross section that influence higher photon scattering at the surface and across inside a layer. The excitation of LSPPs was suitable for applications in the fabrication of lowcost solar cells, photo diodes and optical bio-sensing applications.

\section{Data AvailabiLity Statement}

The data that support the findings of this study are available from the corresponding author upon reasonable request.

\section{REFERENCES}

Barnes, L., William, J., Alain, P. K., Dereux, S., Thomas W., and Ebbesen, W. (2003). "Surface Plasmon subwavelength optics." Nature, 424: 6950: 824.

Billaud, P. (2007). "Optical extinction spectroscopy of single silver nanoparticles." The European Physical Journal D, 43.1-3: 271-274. 
Cao, J., Sun, T. and Grattan, K. T. V. (2014). Gold nanorod-based localized surface Plasmon resonance biosensors: A review. Sensors and Actuators, B: Chemical, 195: 332-351. doi:10.1016/j.snb.2014.01.056.

Catchpole, K., Albert, S. and Polman. R. (2008). "Plasmonic solar cells." Optics express 16.26: 21793-21800.

González, J., Rondano, F. and Barba-Ortega, J. (2015). "Influence of both quantum well thickness and radius on density exciton states on a semiconductor nanoribbon", Journal of Physics: Conference Series, IOP Publishing, pp. 012081.

Hu, M. J., Chen, Z.-Y., Li, L., Au, G.V., Hartland, X., Li, M., Marquez, Y. Xia, (2006), Gold nanostructures: engineering their plasmonic properties for biomedical applications, Chemical Society Reviews, 35: 1084-1094.

Kumar, S. N., Wittenberg, J., and Oh, S. H. (2012). "Nanopore-induced spontaneous concentration for optofluidic sensing and particle assembly," Analytical chemistry, Vol. 85, no. 2, pp. 971-977.

Mansoor, R., Riyadh, Q., Amin Habbeb, R, and AL-Khursan, P. (2018). "Numerical modeling of surface plasmonic polaritons." Results in Physics, 9: 1297-1300.

Nasser, Z.H. (2017)."Synthesis and Characterization Thin Films of $\mathrm{TiO}_{2} \mathrm{Nanorods}$ to Fabrication Hybrid Solar Cell ", Physics department, Basrah University, pp. 190.

Pattnaik, S. P., and Priyabrata., T. K. (2005), "Surface Plasmon resonance." Applied Biochemistry and Biotechnology, 126.2: 79-92.

Petryayeva, E. and Krull, J. (2011). Localized surface Plasmon resonance: nanostructures, bioassays and biosensing - a review, Analytica Chimica Acta, 706: 8-24.

Ritchie, R. H. (1957). "Plasma Losses by Fast Electrons in Thin Films," Physical Review, Vol. 106, pp. 874-881.

Tokman, D. E., Westerhof, M. and Gavrilova, M. A. (2000). "Wave power flux and ray-tracing in regions of resonant absorption." Plasma physics and controlled fusion, 42: 91.

Ungureanu, C., Rayavarapu, R. G., Manohar, S., \& Leeuwen, van, T. G. (2009). Discrete dipole approximation simulations of gold nanorod optical properties: choice of input parameters and comparison with experiment. Journal of Applied Physics, 105: (10), 102032-1/7. [102032]. DOI: $10.1063 / 1.3116139$.

Willets, K. A. and Van Duyne R. P. (2007). Localized surface Plasmon resonance spectroscopy and sensing, Annual Review of Physical Chemistry, Annual Reviews, Palo Alto, 58: 267-297.

Wood, R. W. (1902). “On a Remarkable Case of Uneven Distribution of Light in a Diffraction Spectrum," Proceedings of the Physical Society of London, Vol. 18, p. 269.

Zayats, K., Anatoly V., Igor, I. and Smolyaninov. V. (2003). "Near-field photonics: surface Plasmon polaritons and localized surface plasmons." Journal of Optics A: Pure and Applied Optics 5.4: S16.

$$
--0-
$$

\section{How to cite this article}

Mosiori, C. O., Njoroge, W. K., \& Ochoo, L. O. (2020). Influence of Localized Surface Plasmon Polaritons on Silver Nanoparticles. ABC Journal of Advanced Research, 9(1), 3944. https://doi.org/10.18034/abcjar.v9i1.503 